\title{
Analysis of Trapped Quantum Degenerate Dipolar Excitons
}

\author{
Ronen Rapaport, Gang Chen, and Steven Simon \\ Bell Laboratories, Lucent Technologies, 600 Mountain Avenue, Murray Hill, New Jersey 07974
}

\begin{abstract}
The dynamics of quantum degenerate two-dimensional dipolar excitons confined in electrostatic traps is analyzed and compared to recent experiments. The model results stress the importance of artificial trapping for achieving and sustaining a quantum degenerate exciton fluid in such systems and suggest that a long-lived, spatially uniform, and highly degenerate exciton system was experimentally produced in those electrostatic traps.
\end{abstract}

Excitons are excellent candidates for producing BoseEinstein (BE) quantum statistical effects, such as condensation and superfluidity, on a semiconductor platform [1, 2]. Observation of such effects can open up new and exciting opportunities for both fundamental and applied research. A crucial step in this direction is the ability to obtain long-lived quantum degenerate excitons, which practically means sustaining a high density exciton cloud for times long enough for the excitons to cool down to the lattice temperature. One of the more promising twodimensional (2D) exciton systems for this purpose is the dipolar (or "indirect") exciton system in double quantum well (DQW) structures. In this system, the constituent electrons and holes reside in difference quantum wells, giving the excitons very long recombination lifetimes (in the microsecond range) which should be much longer than their thermalization time with the host lattice. In addition to being able to cool the excitons, to observe BE effects, one must also be able to obtain high densities. Unfortunately, the combined task of cooling an exciton fluid while maintaining high density has turned out to be quite difficult, as recent studies have revealed [3, 4] : the inherent strong dipolar repulsion between pairs of excitons, while desirable for eliminating the formation of eh droplets and other complexes, also leads to a rapid spatial expansion of the initially dense exciton cloud. As a result, the exciton density quickly drops to below the critical value for quantum degeneracy. In addition, the strong exciton expansion prevents the formation of a static fluid and the dipolar repulsion energy is converted into exciton kinetic energy during the expansion, which can result in a heating source which slows the cooling process. This fast expansion of the exciton cloud thus poses a serious technical problem to producing BE effects in exciton systems.

One possible solution is to continuously supply cold dipolar excitons, using long distance in-plane charge transport ("exciton rings") as a cooling mechanism 5, 6, 7]. Recently we have been trying to find a solution by implementing spatially confining potential traps for the dipolar excitons in the plane of the quantum wells, an approach that is somewhat analogous to that of atom trapping and cooling. Two different trapping methods have been attempted experimentally: strain-induced [8] and electrostatic 9, 10, 11] traps (Xtraps). The Xtraps, the design of which is given in Ref. [9] seem to be particularly promising as they are easily tunable in size and depth and have sharp boundaries, all of which are important for achieving high exciton densities, as was recently demonstrated experimentally [10].

In light of these recent experiments, it is important to analyze and understand the exciton dynamics in such Xtraps and their critical role in achieving a long lifetime and spatially uniform degenerate fluid of excitons. The calculations in this paper are based on the models developed by Ivanov et. al. [12, 13], and extended to include the Xtrap potential and expansion induced exciton heating. They explain our previous experimental observations and reveal the detailed dynamics of the dipolar exciton fluid confined in a trap. In particular, these calculations show the advantages of this trapping method, and suggest that the exciton fluid confined in the Xtraps is highly degenerate and spatially uniform, over long time periods of the order of the exciton lifetime.

The inset of Fig. 1 shows an electrostatic trap design 9], where dipolar excitons (with a dipole moment given by $\overrightarrow{d_{X}}=-e z_{0} \hat{z}, z_{0}$ being the effective dipole length) are trapped under a local circular electrostatic gate with radius $R$. When an electrical bias is applied to the gate, the (negative) dipole-field interaction energy profile $\varepsilon_{t}$ at the DQW position, plotted in Fig. 1 (solid line), effectively confines the excitons to the region under the circular gate with the trapping force given by $\mathbf{F}_{t}=-\nabla \varepsilon_{t}$ [9]. For calculation convenience, we approximate (Fig. 1] dashed line) the xtrap potential with an analytic function: $\varepsilon_{t}(r)=\frac{\varepsilon_{t}^{0}}{2}\left(1+\tanh \frac{2(r-R)}{\delta}\right)$. Here, $\varepsilon_{t}^{0}$ is the energy at the trap center, which is proportional to the applied voltage, and $\delta$ is the effective "thickness" of the boundary region, proportional to the distance between the the top gate and bottom electrode.

Two scattering mechanism for dipolar excitons are included in this model. The first one is the fast, density dependent exciton-exciton scattering, which is dominant at high exciton densities. Such a scattering process, with a characteristic time much smaller than any other time scale of the dynamics, yields internal exciton equilibrium and therefore a well defined exciton temperature, $T_{X}$. It is also responsible for a density dependent diffusion coefficient, $D_{X X}$, calculated by Ivanov et al. in Ref. [12]. 


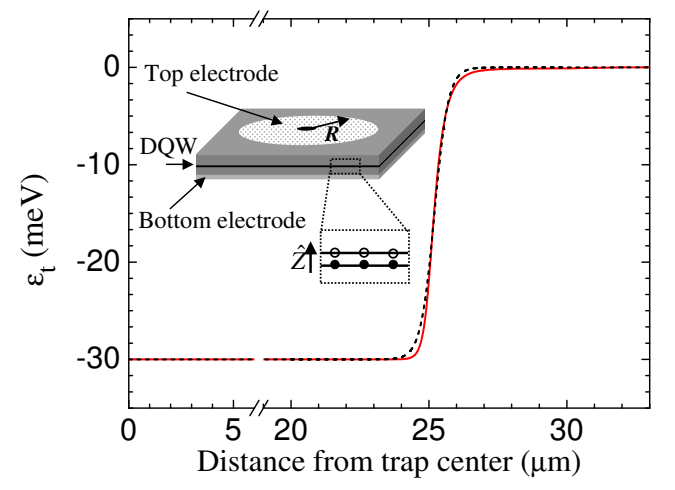

FIG. 1: The calculated Xtrap potential radial profile (solid line) and its analytic approximation (dashed line). The inset shows an schematic diagram of an Xtrap design geometry.

The other scattering mechanism is due to exciton-QW disorder interaction, which is density independent but yields a diffusion coefficient, $D_{d o}$, which depends on the QW width to the sixth power (see Refs. [3, 4]). We also assume that there is a single characteristic time $\left(\tau_{l}\right)$ for the thermalization of the exciton gas with the lattice (with a lattice temperature $T_{l}$ ). The dynamics of a dipolar exciton fluid in an Xtrap can then be described by two coupled equations for the exciton fluid density and temperature. The first nonlinear diffusion equation describes the time evolution of the exciton fluid:

$$
\frac{\partial n_{X}}{\partial t}+\nabla \cdot\left(\mathbf{J}_{D}+\mathbf{J}_{d}+\mathbf{J}_{t}\right)+\frac{n_{X}}{\tau_{X}}-I_{X}(r, t)=0 .
$$

Here, $n_{X} \equiv n_{X}(\mathbf{r}, t)$ is the exciton density profile. $\mathbf{J}_{D}$, $\mathbf{J}_{d}$, and $\mathbf{J}_{t}$ are the three exciton currents which are driven by diffusion, dipolar repulsion, and the trapping potential, respectively. $I_{X}(r, t)=I_{X}(r) \delta(t)$ is the exciton source for a short optical pulse excitation and $\tau_{X}$ is the lifetime of the excitons, which is assumed to be density independent. These exciton currents are related to the various forces through the exciton mobility $\mathbf{J}=n_{X} \mu \mathbf{F}$. The exciton mobility, $\mu$, is related to the effective exciton diffusion coefficient $D=D_{X X} D_{d o} /\left(D_{X X}+D_{d o}\right)$, through Einstein relations $\mu=\left(D / k T_{0}\right)\left(e^{T_{0} / T_{X}}-1\right)$, with $T_{0}=\left(2 \pi \hbar^{2} n_{X}\right) /\left(\mathrm{kgm}_{X}\right)$ being the degeneracy temperature. Here, $m_{X}=0.2 m_{e}$ is the exciton mass and $g=4$ is the exciton spin degeneracy. The dipole-repulsion force, $\mathbf{F}_{d}=-\alpha \nabla n_{X}$ is due to the mean field dipoledipole interaction energy [14]: $\varepsilon_{d d}=4 \pi e d_{X} n_{X} / \epsilon \equiv \alpha n_{X}$, where $\epsilon$ is the background dielectric constant. The diffusive force is $\mathbf{F}_{D}=-\nabla \zeta=-\frac{k T_{0} / n_{X}}{e^{T_{0} / T_{X}-1}} \nabla n_{X}$, where $\zeta=k T_{X} \ln \left(1-e^{-T_{0} / T_{X}}\right)$ is the chemical potential in the non-interacting limit. The trapping force for the dipolar excitons can be derived from the above trapping potential: $\mathbf{F}_{t}=-\frac{\varepsilon_{t}^{0}}{\delta}\left[1-\tanh ^{2} \frac{2(r-R)}{\delta}\right] \hat{\mathbf{r}}$. The three expressions for the exciton currents in Eq. (10) are there- fore: (a) $\mathbf{J}_{d}=-\mu \alpha n_{X} \nabla n_{X}$, (b) $\mathbf{J}_{D}=-D \nabla n_{X}$, and (c) $\mathbf{J}_{t}=-\mu n_{X} \frac{\varepsilon_{t}^{0}}{\delta}\left[1-\tanh ^{2} \frac{2(r-R)}{\delta}\right] \hat{\mathbf{r}}$.

The second equation describes the time evolution of the exciton fluid temperature, $T_{X}$ :

$$
\frac{d T_{X}}{d t}=-\left[\frac{1}{k_{B} N_{X}}\left(\frac{\partial E_{d d}}{\partial t}\right)_{N_{X}}+\frac{T_{X}-T_{l}}{\tau_{l}}\right],
$$

where $E_{d d}$, the total potential energy due to the dipoledipole interactions is given by: $E_{d d}=\int \alpha n_{X}^{2}(\mathbf{r}, t) d^{2} r$ and $N_{X}(t)=\int n_{X}(\mathbf{r}, t) d^{2} r$ is the total number of dipolar excitons. The first term on the RHS of Eq. 2 represents the heating of the exciton gas due to the driven expansion and the last term represents the thermalization of the excitons with the lattice.

Eqs.(11),(2) are coupled and can be numerically evaluated. For the following calculations, we use parameters that are typical for our experiments with GaAs double QW structures 10. The size of the trap is taken as $R=25 \mu \mathrm{m}$, consistent with the experiments performed in [10]. The depth of the trap is fixed at $\varepsilon_{t}^{0}=30 \mathrm{meV}$. For a short $(\sim 1 n s)$ pulse excitation with a gaussian profile, we assume that dipolar excitons are subsequently created at the center of the trap with an initial gaussian profile: $n_{X}(r, t=0)=n_{0} e^{-r^{2} / w_{0}^{2}}$ where typically for our experiments, $w_{0}=15 \mu \mathrm{m}$ and $n_{0}=1.5 \times 10^{11} \mathrm{~cm}^{-2}$. The initial exciton temperature is taken to be the energy difference between the optical excitation energy and the dipolar exciton energy, $T_{X}(t=0) \simeq 50 K$, which is much hotter than the lattice temperature $T_{l}=1.4 K$, as we assume that the optically excited carriers rapidly transform into hot dipolar excitons. The exciton lifetime, $\tau_{X}$ is position dependent. Inside the trap, the excitons are dipolar and their lifetime is in microsecond range. For the particular trap depth $30 \mathrm{meV}$, we use a typical value of $\tau_{X}=\tau_{X_{I D}}=1.5 \mu \mathrm{s}$. Outside the trap, the excitons are direct and their lifetime is taken as $\tau_{X}=\tau_{X_{D}}=0.1 \mathrm{~ns}$. In the trap boundary region, the dipolar excitons are subject to ionization due to the in-plane fringing fields that depends on the geometry of the trap design (see Ref. 9] for details). This ionization process yields an effective boundary lifetime for the excitons $\left(\tau_{\text {trap }}\right)$. We have shown analytically [9] that this lifetime, and thus the right Xtrap design, is crucial for the effectiveness of the Xtrap, as was further confirmed by our experiments [10] and the following calculations.

Fig. 2 plots the radial density profile of the excitons in a high quality Xtrap with negligible boundary ionization, in which case the boundary lifetime is only limited by the intrinsic lifetime of the excitons $\left(\tau_{\text {trap }}=\tau_{X}\right)$. The values of the diffusion coefficients are chosen by fitting the expansion dynamics (the cloud FWHM as a function of time in particular) of a free expanding excitons to experimental results (see Ref. 3] ). In a good agreement with the experimental results of Ref. 10], within tens of nanoseconds the profile flattens, as the driven expan- 


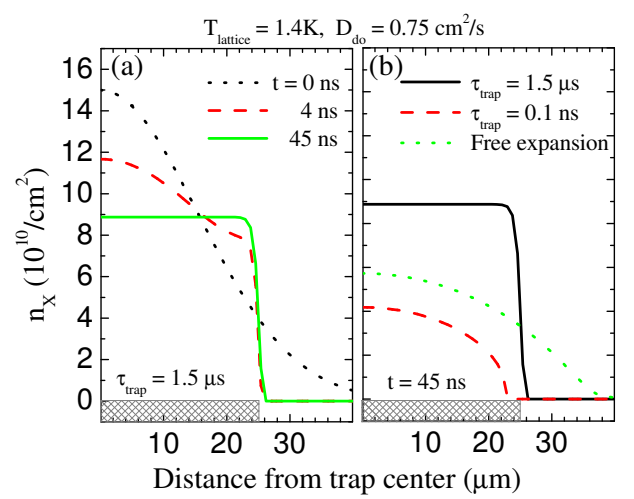

FIG. 2: Radial profile of the exciton density distribution for (a) a high quality Xtrap $\left(\tau_{\text {trap }}=1.5 \mu \mathrm{s}\right)$ at various times, and (b) High quality Xtrap $\left(\tau_{\text {trap }}=1.5 \mu \mathrm{s}\right.$, solid line), low quality Xtrap $\left(\tau_{\text {trap }}=0.1 n s\right.$, dashed line $)$, and free expanding excitons (dotted line) at $t=45 \mathrm{~ns}$. The bar at the bottom of each figure mark the radial extent of the Xtrap.

sion by the dipole repulsion pushes the excitons to the reflecting trap boundary. These two competing forces result in a stable flat exciton density profile (the density profile with the lowest total energy) that decays with the characteristic radiative lifetime of the excitons, $\tau_{X}$. As we will show below, as the profile flattens out, the exciton quickly cools to the lattice temperature and becomes highly degenerate. This degeneracy is maintained on the time scale of the exciton radiative lifetime.

This result is strikingly different from either a free expanding exciton cloud or excitons in a low quality Xtrap with a considerable boundary ionization, as depicted in Fig. 2 2 b. In the case of free expanding excitons, the density rapidly decreases, as the driven expansion continues to spread the exciton cloud to large radii. For a low quality trap $\left(\tau_{\text {trap }}=0.1 n s\right)$, excitons reaching the trap boundaries are effectively eliminated by ionization, rapidly depleting the expanding exciton cloud. Note that in this case, the exciton profile remains curved and does not flattens like the exciton pool in the high quality trap. This is also in agreement with the experimental profile found for a low quality Xtrap in Ref. 10.

To see the dramatic effect of trapping on high mobility excitons $\left(D_{d o}=10 \mathrm{~cm}^{2} / \mathrm{s}\right)$, we show the calculated ground state occupation number of the 2D excitons, $N_{E=0}=\left(\exp \left(T_{0} / T_{X}\right)-1\right)$, as a function of time after photoexcitation, for excitons in a high and low quality Xtrap as well as for free excitons. Initially, the ground state occupation is much smaller than unity, as the excitons are hot and essentially classical. The exciton cloud then start to expand and cool. As the transition to a distinctive $\mathrm{BE}$ statistics arises when $T_{0} / T_{X} \sim 1$, there is a competition between cooling, that tend to increase $N_{E=0}$ and expansion that tend to decrease it due to the drop of $n_{X}$. In a high quality Xtrap, expansion is lim-

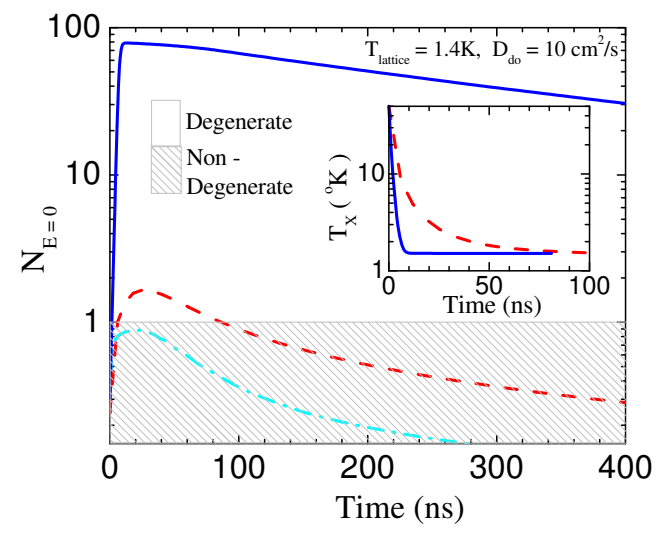

FIG. 3: The exciton ground state occupancy, $N_{E=0}$, as a function of time for a high quality Xtrap $\left(\tau_{\text {trap }}=1.5 \mu \mathrm{s}\right.$, solid line), a low quality trap $\left(\tau_{\text {trap }}=1 n s\right.$, dotted line $)$ and free expanding excitons (dashed line). The inset shows the corresponding exciton temperature as a function of time for the high quality Xtrap (solid line) and free expending excitons (dotted-dashed line).

ited to the Xtrap boundaries, and thus reaches steady state after $\sim 10$ nanoseconds. Cooling is then efficient (as is seen in the inset of Fig. 3) and the excitons reach a stable high degenerate state with $N_{E=0} \gg 1$, that decays slowly on the time scale of $\tau_{X}$. On the contrary, if the excitons are freely expanding (no trap), their density continues to drop as they expand. While they continue to cool by interaction with the lattice, the expansion itself heats up the cloud, as more internal potential energy is lost and converted into heat by the fast exciton-exciton interaction. This leads to a slower net cooling rate for the free expanding excitons compared with the trapped ones, and consequently the excitons reach only marginal quantum degeneracy before their density drops too low due to the fast driven expansion. Finally, in a low quality trap, the drop in exciton density due to boundary ionization dominates over the cooling, completely preventing the excitons from reaching degeneracy.

While those calculations strongly suggest that the exciton pool that was created in Ref. 10 is highly degenerate for hundreds of nanoseconds, it is based on the assumption that the cooling rate is indeed much shorter than the exciton lifetime. While this is a very reasonable assumption (see Ref. [12]), a clear experimental evidence of this degeneracy that is independent on model assumptions is still essential, and experiments looking for such conclusive evidence are ongoing.

In summary, the dynamics of two-dimensional dipolar excitons confined in electrostatic traps is modeled and agrees well with recent experiments. The model shows that artificial trapping is crucial for achieving and sustaining a quantum degenerate exciton fluid is such systems and suggest that a spatially uniform, highly degen- 
erate exciton pool was experimentally produced in those electrostatic traps.

[1] D. Snoke, Science 298, 1368 (2002).

[2] J. P. Eisenstein and A. H. MacDonald, Nature 432691 (2004).

[3] R. Rapaport, G. Chen, and S. Simon, Phys. Rev. B 73, 033319 (2006).

[4] Z. Vörös, R. Balili, D.W. Snoke, L. N. Pfeiffer, and K. West, Phys. Rev. Lett. 94, 226401 (2005).

[5] L. V. Butov et al., Phys. Rev. Lett. 92, 117404 (2004).

[6] R. Rapaport et al., Phys. Rev. Lett. 92, 117405 (2004).
[7] S. Yang, A. T. Hammack, M. M. Fogler, and L. V. Butov, cond-mat/0606683 (2006).

[8] D. Snoke, Y. Liu, Z. Voros, L. N. Pfeiffer, and K. West, Solid State Commun. 134, 37 (2005).

[9] R. Rapaport et al., Phys. Rev. B, 72, 075428 (2005).

[10] G. Chen, R. Rapaport, L. N. Pfeiffer, K. West, P. M. Platzman, S. Simon, Z. Voros, D. Snoke, Phys. Rev. B 74, 045309 (2006), Condmat/0601719 (2006).

[11] A. T. Hammack et al., Condmat/0504045 (2005).

[12] A. L. Ivanov, P. B. Littlewood, H. Haug, Phys. Rev. B, 595032 (1999).

[13] A. L. Ivanov, Europhys. Lett., 59586 (2002).

[14] S. Ben-Tabou de-Leon and B. Laikhtman, Phys. Rev. B 63, 125306 (2001). 